\title{
Transition to Turbulence for a Quartz Tuning Fork in Superfluid ${ }^{4} \mathrm{He}$
}

\author{
D.I. Bradley, M.J. Fear, S.N. Fisher, \\ A.M. Guénault, R.P. Haley, C.R. Lawson, \\ P.V.E. McClintock, G.R. Pickett, R. Schanen, \\ V. Tsepelin and L.A. Wheatland
}

Received: date / Accepted: date

\begin{abstract}
We have studied the resonance of a commercial quartz tuning fork immersed in superfluid ${ }^{4} \mathrm{He}$, at temperatures between $5 \mathrm{mK}$ and $1 \mathrm{~K}$, and at pressures between zero and 25 bar. The force-velocity curves for the tuning fork show a linear damping force at low velocities. On increasing velocity we see a transition corresponding to the appearance of extra drag due to quantized vortex lines in the superfluid. We loosely call this extra contribution "turbulent drag". The turbulent drag force, obtained after subtracting a linear damping force, is independent of pressure and temperature below $1 \mathrm{~K}$, and is easily fitted by an empirical formula. The transition from linear damping (laminar flow) occurs at a well-defined critical velocity that has the same value for the pressures and temperatures that we have measured. Later experiments using the same fork in a new cell revealed different behaviour, with the velocity stepping discontinuously at the transition, somewhat similar to previous observations on vibrating wire resonators and oscillating spheres. We compare and contrast the observed behaviour of the superfluid drag and inertial forces with that measured for vibrating wires.
\end{abstract}

Keywords Superfluid · Turbulence Critical velocity · Tuning fork

PACS $67.25 . \mathrm{dk} \cdot 67.25 . \mathrm{dg} \cdot 47.27 . \mathrm{Cn}$

\section{Introduction}

There is much interest in the behaviour of vibrating objects immersed in the quantum fluids ${ }^{3} \mathrm{He}$ and ${ }^{4} \mathrm{He}$. The recent expansion in activity has been largely inspired by developments in the study of turbulence, with several groups investigating oscillating wires [1-12], grids [13-18], spheres [19-25] and quartz tuning forks [26-33]. Mechanical probes give much information about the dynamics of superfluids and their excitations. Measurements of the resonant frequency provide information on the inertial backflow around the object, while the damping measures dissipation of the superfluid and/or

R.P. Haley

Department of Physics, Lancaster University, UK.

Tel.: +44-1524-593224

Fax: +44-1524-844037

E-mail: r.haley@lancaster.ac.uk 
normal fluid components. Vibrating objects are therefore very sensitive to the production of quantised vortices in a superfluid. The complex evolution of a tangle of such vortices has become commonly known as "quantum turbulence". Vibrating objects are also widely used as secondary thermometers by measuring damping due to the thermal excitations [34-38].

Quantum turbulence, particulary in the low temperature limit, is conceptually much simpler than classical turbulence, being composed of a tangle of similar quantised vortices, moving in an ideal (inviscid and almost incompressible) fluid according to their self-induced superfluid flow. The relative simplicity of quantum turbulence also facilitates the development of theoretical models and computer simulations. Much of the recent experimental work on quantum turbulence from vibrating structures has been complimented by detailed numerical simulations [39-45] and theoretical work [4650]. Many of the recent developments can also be found in review articles such as [51, $52]$.

In superfluid ${ }^{3} \mathrm{He}$ advances in experimental techniques have enabled studies of the production and decay of turbulence by vibrating wires and grids $[4,5,13-16]$. In superfluid ${ }^{4}$ He the turbulent damping of an oscillating grid has been probed $[17,18]$, and studies of spheres [19-22] and vibrating wires [1,2,6-11] show common features such as hysteresis around the turbulent transition, likely related to the properties of remnant vortices. Recent attention has been given to the production of quantum turbulence by quartz tuning forks $[28,31,33]$. Here we concentrate on the transition to turbulence in superfluid ${ }^{4} \mathrm{He}$ at low temperatures using a commercially available quartz tuning fork.

We have investigated the properties of a tuning fork in superfluid ${ }^{4} \mathrm{He}$ over the whole range of pressure, from zero bar to the melting curve, and at temperatures from $1 \mathrm{~K}$ down to $\sim 5 \mathrm{mK}$, using a dilution refrigerator. We have measured the transition from laminar to turbulent flow by measuring force-velocity curves at the resonant frequency of the fork. At the lowest temperatures, where the ${ }^{4} \mathrm{He}$ is virtually all superfluid, the transition from linear damping in the laminar regime to non-linear turbulent damping occurs at a well defined critical velocity. At higher temperatures the transition is less clear, somewhat masked due to increased linear damping. Nevertheless, the turbulent drag force is found to be independent of temperature below $1 \mathrm{~K}$. In further investigations of the transition region at low temperatures, we find hysteresis and switching behaviour similar to that observed for vibrating wires and spheres [1,2,6-11,19-22]. There are however some important differences which we discuss below.

\section{Experimental}

Our experimental cell is illustrated in figure 1(a). The quartz tuning fork shown in figure 1(b) is commercially available [53] and made to operate close to $2^{15}=32768 \mathrm{~Hz}$ in ambient conditions. Each of the two prongs has length $L=3340 \mu \mathrm{m}$, width $W=$ $300 \mu \mathrm{m}$ and thickness $T=450 \mu \mathrm{m}$. The distance between the prongs is $260 \mu \mathrm{m}$.

The cylindrical cell body is machined from Araldite epoxy resin. It contains silver sinter heat exchangers, which are connected by a high conductivity silver wire to a sinter pad in the mixing chamber of our dilution refrigerator. The cell is filled through a narrow capillary which is heat sunk at the pot, the still and the cold plate. The ${ }^{4} \mathrm{He}$ is so-called "technical helium", collected from a transport dewar. The cell is mounted on the cryostat so that the prongs of the fork are horizontal, in an effort to prevent problems with "dirt" falling onto the fork. We have removed only the top of the metal 
(a)

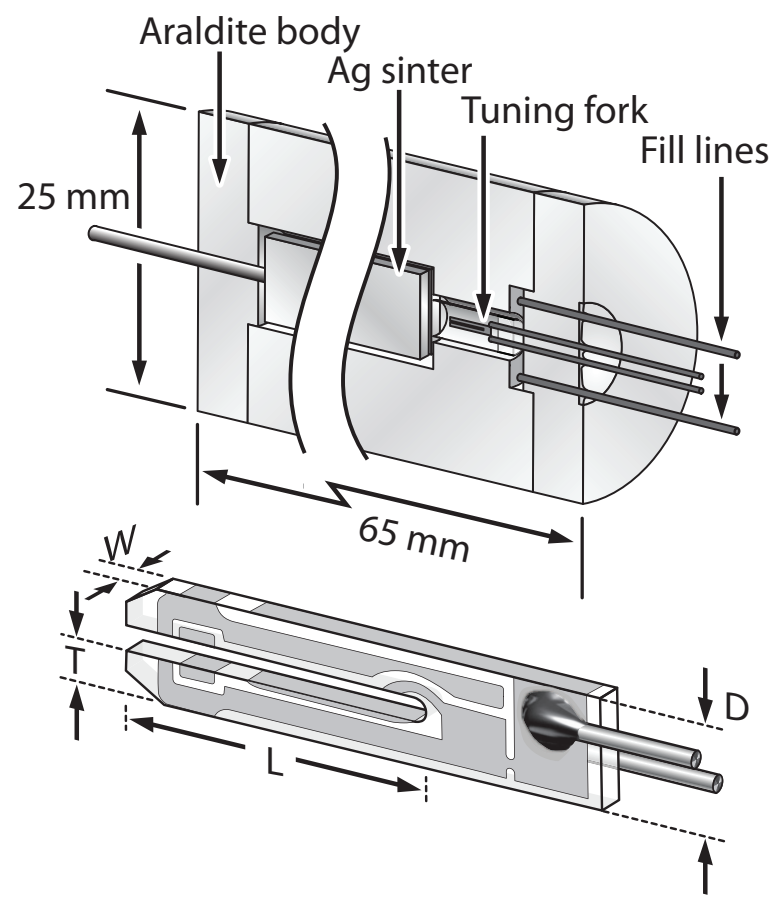

Fig. 1 Schematic of (a) the experimental cell and (b) quartz tuning fork. The fork is encased by a metal can which is $3 \mathrm{~mm}$ in diameter. The top of the can has been cut away to allow the helium to enter. The fork dimensions were measured with a scanning electron microscope: $L=3340 \mu \mathrm{m} ; T=450 \mu \mathrm{m} ; W=300 \mu \mathrm{m} ; D=1160 \mu \mathrm{m}$; prong separation $260 \mu \mathrm{m}$.

packaging can in which the fork is supplied, and the original electrical leads are glued through the end of the cell.

The mixing chamber temperature is monitored with a vibrating wire resonator in the dilute phase below $50 \mathrm{mK}$. Above $50 \mathrm{mK}$ a carbon resistance thermometer is used to measure the refrigerator temperature. There is no thermometry in the cell itself. Measurements were made while the fridge was held at a given temperature, and after the cell had come into thermal equilibrium, as determined by when the response of the fork had stabilized. This procedure was adequate for our purposes, since at higher temperatures thermal gradients should not be significant, and at lower temperatures the fork response is quite insensitive to temperature.

The fork is driven by applying an AC voltage to its electrodes. This exerts a driving force on the prongs of the tuning fork due to the piezoelectric properties of quartz. As the prongs move, an electric current is generated proportional to their velocity. Thus we can infer the velocity response of the prongs as a function of the driving force by measuring the current produced as a function of the applied voltage.

The driving voltage is produced by a waveform generator, with suitable attenuation as required. The response current is measured using a calibrated custom-made currentto-voltage converter [54] at the input of a lock-in amplifier. We have measured the properties of the fork using a variety of techniques. 
A "frequency sweep" involves sweeping the frequency of the driving voltage, at constant amplitude, and measuring the subsequent current. A dual channel phase sensitive lock-in amplifier is used, and we record both components of the current, in-phase and out-of-phase with the driving voltage. The two components are fitted to the ideal Lorentzian lineshape, expected for a linear oscillator. The fit also includes a linear background current, arising from the electrical properties of measurement circuit. The frequency sweeps, in the linear response region, allow us to characterize the resonance accurately, to fine-tune the phase offset of the lock-in amplifier, and to determine the background current, the resonant frequency $f_{0}$, the resonant height $I_{0}$ and the resonance frequency width $\Delta f_{2}$, measured at the half-height points of the in-phase response.

To measure the force-velocity response of the fork, we use a "drive sweep". Here, we sweep the driving voltage at the resonance frequency and measure the resulting current. We use a tracking routine to adjust the frequency of the driving voltage during the drive sweep, in order to maintain resonance. The frequency is adjusted continuously to minimise the out-of-phase current, a procedure which, in practice, very closely corresponds to the maximum (in-phase) signal current, and hence to maximum power dissipation. The out-of phase signal current is very sensitive to small changes in frequency away from the resonant frequency, so this allows an accurate measurement of any changes in the resonant frequency.

We relate the measured electrical properties of the fork to its mechanical properties using the convention used by other groups, following [55] and as detailed in [26]. The current $I$ produced by the tuning fork motion is related to the velocity $v$ of the tip of the prongs via a fork constant $a$ :

$$
I=a v
$$

The fork constant also determines the relation between driving force $F$ and applied voltage $V$ :

$$
F=\frac{a V}{2} .
$$

This is evident from energy considerations. The product of force and in-phase velocity is the power absorbed, which is equal to the product of applied voltage and the inphase current. The factor of two arises since the fork has two prongs which move in anti-phase.

As discussed [26], we can determine $a$ from the "height-times-width-over-drive" of the Lorentzian resonance, since

$$
\frac{I_{0} \Delta f_{2}}{V_{0}}=\frac{a^{2}}{4 \pi m}
$$

Here, $I_{0}$ is the (in-phase) current amplitude at resonance, $V_{0}$ is the applied voltage amplitude and $m$ is the effective mass of a prong. The value of $I_{0} \Delta f_{2} / V_{0}$ is most readily obtained from a frequency sweep in the linear response regime at low drives. The effective mass in vacuum, calculated for an ideal bending beam with a rectangular cross section, is found to be 0.24267 times the actual mass of a prong [26,55], i.e.

$$
m=0.24267 \rho_{q} L W T
$$

where $\rho_{q}=2659 \mathrm{~kg} / \mathrm{m}^{3}$ is the density of quartz. The errors involved in this procedure to determine the absolute velocity and force on each prong are unknown, but probably on the scale of $10 \%$ or less. We note that in practice the fork constant will depend 
on the detailed geometry of the fork including its electrodes and so may differ slightly from one fork to another. For our fork, we estimate the effective mass to be about $0.30 \mathrm{mg}$ and the constant $a$ to be about $12 \mu \mathrm{C} \mathrm{m}^{-1}$.

\section{Drag and Inertial Coefficients}

The force exerted on an object oscillating in a fluid flow can be expressed in terms of two dimensionless coefficients. The drag coefficient $C_{D}$ describes the dissipative component of the force while the inertial coefficient $C_{M}$ describes the non-dissipative component. We can define the drag coefficient for the fork by the equation

$$
F=\frac{1}{2} C_{D} \rho A v^{2}
$$

where $A=L W$ is the cross-sectional area of each prong perpendicular to the motion, and $\rho$ is the density of the helium. Here, and in the following, we define $v$ to be the velocity amplitude of the tip of the prongs and $F$ to be the amplitude of the driving force as inferred from equations 1 and 2 above. As discussed in [2], for cylinders oscillating in classical fluids the similarly defined $C_{D}$ is of order unity for high velocity turbulent flows, although it has some velocity dependence [56].

Using analogous arguments to those discussed for vibrating wire resonators [2], we can define an inertial coefficient $C_{M}$, as used in the Morison equation [57] for classical fluids. The inertial force per unit length on a cylinder of diameter $D$ can be written as

$$
F=\frac{1}{4} \pi \rho D^{2} C_{M} \frac{d v}{d t}=\delta m \frac{d v}{d t}
$$

where $\rho$ is the fluid density and $\delta m$ is the effective mass per unit length of the fluid back-flow around the cylinder. Since the resonant frequency of the oscillating object is inversely proportional to the square-root of its effective mass, the shift $\delta f$ due to back-flow is

$$
\frac{\delta f}{f}=-\frac{1}{2} \frac{\delta m}{m}=-\frac{1}{2} \frac{\rho}{\rho_{o}} C_{M}
$$

where $f$ is the resonant frequency in vacuum and $\rho_{o}$ is the density of the oscillator material. We use equation 7 to define the inertia coefficient of our fork, with $\rho_{o}=\rho_{q}$, the density of quartz. For an ideal cylinder in pure potential flow, the inertial coefficient is $C_{M}=1$. It is not clear what value we should expect for a tuning fork prong since it has a rectangular cross-section, roughly-shaped corners, and a flow field modified by the proximity of the other prong.

For cylinders oscillating in classical fluids and for vibrating wires in superfluid ${ }^{4} \mathrm{He}$ at low temperatures, $C_{M}$ falls below unity at higher velocities, as the flow becomes turbulent [2].

\section{Results and Discussion}

\subsection{Force-Velocity Curves at Low Temperatures}

Figure 2 shows force-velocity curves at $5 \mathrm{mK}$ inferred from drive sweep measurements as discussed above. At low velocities, below $\sim 50 \mathrm{~mm} \mathrm{~s}^{-1}$, the damping force is very 


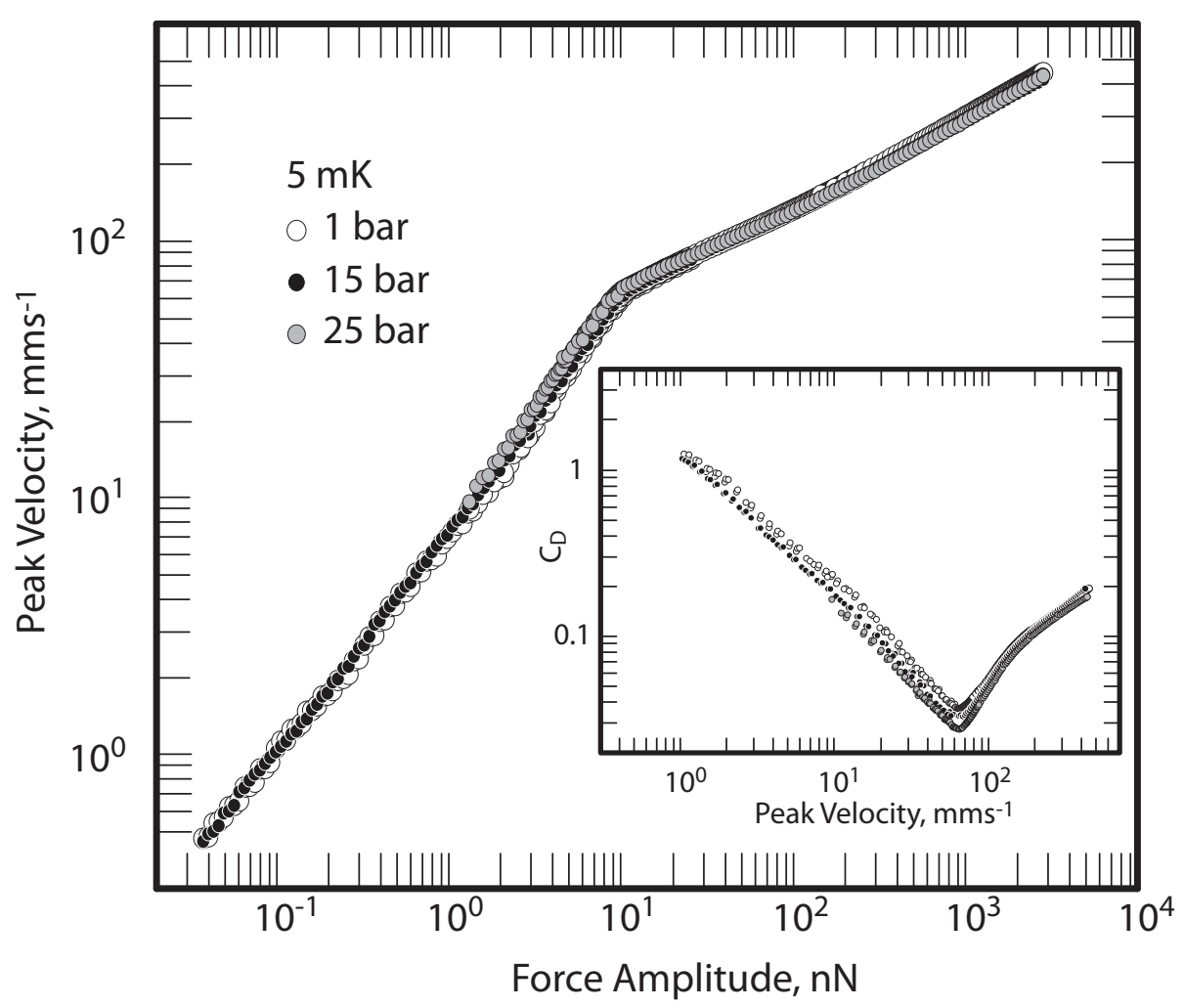

Fig. 2 The response of the tuning fork in superfluid ${ }^{4} \mathrm{He}$ at the lowest temperature, for three different pressures. The inferred velocity amplitude of the tip of the prong (peak velocity) is plotted against the amplitude of the driving force for each prong. Inset shows the derived drag coefficient, $C_{D}$, plotted as a function of the peak velocity, see text.

nearly linear in velocity (equivalent to a Lorentzian resonance lineshape) and $F / v$ gives the damping coefficient which is proportional to the frequency width $\Delta f_{2}$ of the resonance. At these very low temperatures there are virtually no thermal excitations (i.e. no "normal fluid") in the helium, and we believe that the damping corresponds to the intrinsic damping of the fork itself, i.e. the same damping would be measured in vacuum under equivalent conditions. This intrinsic damping gives a resonance width of order $0.03 \mathrm{~Hz}$ (giving a "Q-value" of order $10^{6}$ ) and is actually slightly drive-dependent, leading to the very small deviation from linearity of the curves at low velocities.

As the drive force is increased, the response of the fork changes quite abruptly to a more non-linear behaviour. We associate this with extra drag arising from quantised vortex lines, so we will loosely refer to this as the "turbulent regime" to distinguish it from the "laminar regime" without vortex lines at lower velocities. Similar behaviour has been observed previously for forks $[28,29,31,33]$, vibrating grids $[17,18]$, vibrating wire resonators $[1,2,6-11]$ and oscillating spheres [19-22] in superfluid ${ }^{4} \mathrm{He}$.

Later measurements of the transition region showed that switching between the two regimes and hysteresis may be observed; we discuss this further below. We also note that, intermittently, apparently similar measurements are not so well-behaved, 


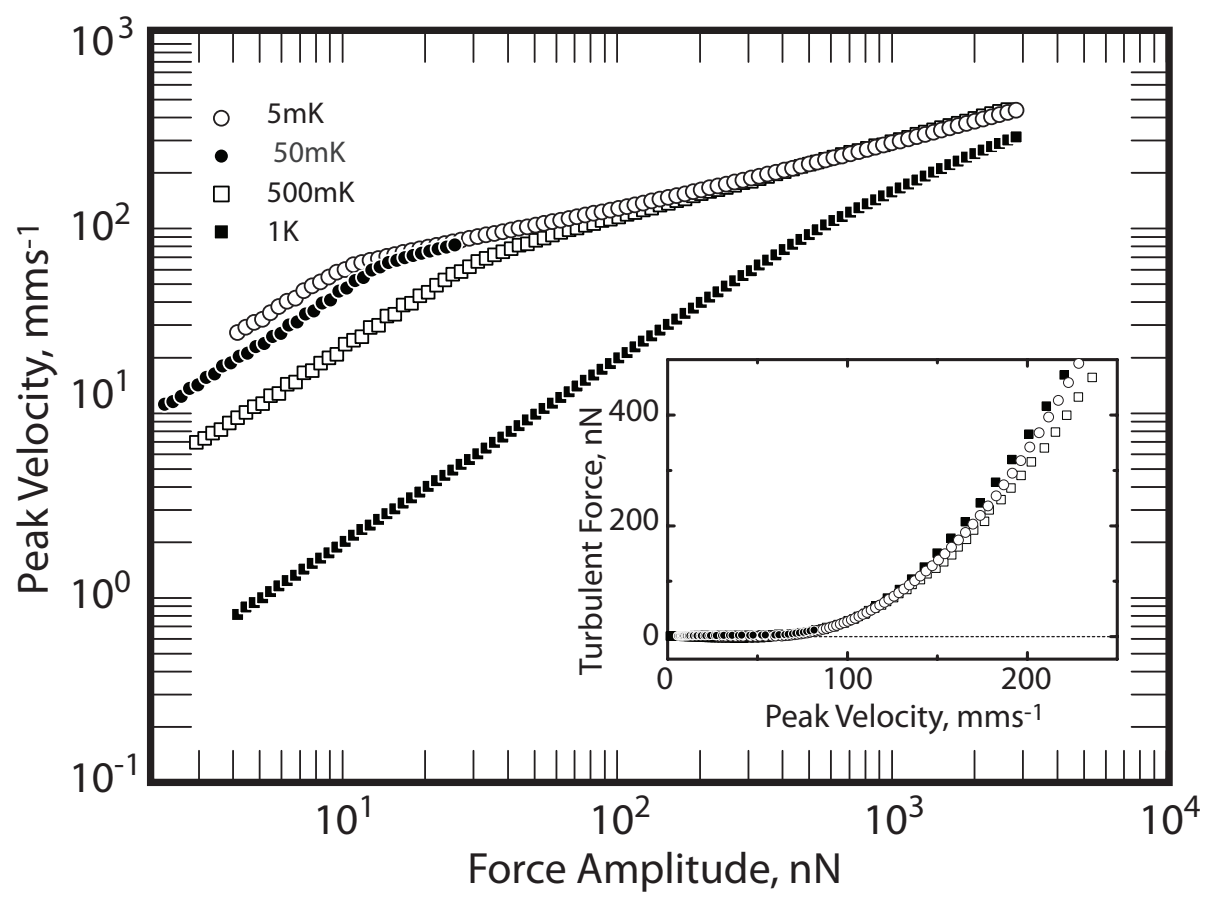

Fig. 3 The force-velocity curves of the same fork at various temperatures, all at 1 bar pressure. The inset shows the same data but with the linear damping behavior subtracted, plotted on a linear scale showing the turbulent drag contribution to the damping force as a function of velocity, and focussing on velocities close to the turbulent transition, see text. (Note that the $50 \mathrm{mK}$ data was only measured up to $85 \mathrm{mms}^{-1}$.)

and we have observed large time-dependent anomalies in the linearly damped region. We believe that some of this anomalous behaviour may be due to dirt, such as small particles of hydrogen in the technical helium that we have used. However, there might also be interesting affects associated with vortex lines pinned between the prongs of the fork.

As revealed in Fig. 2, the force-velocity curves are virtually identical for all pressures, indicating that the turbulent drag at low temperatures has very little, if any, dependence on pressure. At first sight, this might seem surprising given that many of the superfluid properties (governed by the superfluid transition temperature and density) are quite pressure dependent. We note however that the circulation quantum $\kappa=h / m$, which defines the circulation around quantum vortices, is independent of pressure and temperature. Similar observations have been made using a vibrating grid $[17]$.

In the inset to Fig. 2 we show the corresponding drag coefficient $C_{D}$, inferred from equation 5 , as a function of the peak prong velocity $v$. The results are broadly similar to those obtained previously $[28,29,31,33]$. At low velocities the drag is dominated by the intrinsic damping of the fork, giving a roughly $C_{D} \propto v^{-1}$ variation, while at higher velocities there is a sharp cross-over corresponding to the onset of extra drag from quantised vortex lines. Although the data doesn't extend to very high velocities, it is 
consistent with the drag coefficient approaching values of order unity. It is interesting to note that the data appears to show a small pressure dependence when plotted in terms of the drag coefficient. This is due to the pressure dependence of the helium density. Thus, if one associates the lack of a pressure dependence with the pressure independent circulation quantum $\kappa$, then this result would suggest that $\kappa$ is more directly linked to the turbulent drag force than the turbulent drag coefficient. However, since the pressure dependence is small, further measurements are needed to confirm this.

Figure 3 shows the temperature dependence of the force-velocity response curves of the fork from $5 \mathrm{mK}$ up to $1 \mathrm{~K}$ at 1 bar pressure. At the lowest temperatures the near linear damping at low velocities is temperature independent and dominated by intrinsic damping as discussed above. At higher temperatures the damping is due to ballistic phonon scattering, and at even higher temperatures (above $1 \mathrm{~K}$ ) the damping would eventually be described in terms of viscous damping from the normal fluid component [33]. We can remove the effects of the intrinsic damping by simply subtracting a force, proportional to velocity, to leave only the contribution from the vortices. Hence we find a "turbulent drag force"

$$
F_{\text {turb }}(v)=F(v)-\gamma v,
$$

where $\gamma$ is determined from $F / v$ at low velocities. We can apply the same procedure at higher temperatures also, thus removing both the intrinsic and thermal contributions to the damping. The resultant data are plotted in the inset to figure 3. Evidently all the curves collapse onto a single line. This clearly implies that the turbulent drag force at low temperatures is temperature independent, and that it is independent of the thermal damping force from ballistic phonons. This property was also suggested by measurements reported in [33]. We further note that this contrasts with the behaviour in a classical fluid, where the contributions from laminar and turbulent flows are not additive [58].

In figure 4 the data of figure 3 are re-plotted to show the drag coefficient as a function of the peak velocity. At higher velocities the drag coefficient appears to be leveling towards a value of order unity. Unfortunately our measurements do not extend to higher velocities since we did not want to risk destruction of the fork (at velocities around $1-2 \mathrm{~m} \mathrm{~s}^{-1}$ the prongs are being shaken so violently that they may break). The inset to figure 4 shows the turbulent drag coefficient, derived from the turbulent drag force defined by equation (8). For all the superfluid data, there is a clear transition to turbulent drag at a critical velocity $v_{c} \simeq 63 \mathrm{~mm} \mathrm{~s}^{-1}$. We note that the $500 \mathrm{mK}$ data in Figs. 3 and 4 appears to deviate slightly at higher velocities from the trend observed at lower and higher temperatures. The reason for this is not known, but it may due to thermal drift; we were unable to keep the fridge temperature very stable in this temperature range.

We have fitted the data in Fig. 4 to an empirical expression given by

$$
C_{D}=\frac{\alpha}{v}+\beta H\left(v-v_{c}\right) \frac{\left(v^{2}-v_{c}^{2}\right)}{v^{2}}\left(\frac{\left(\frac{v}{v_{c}}-1\right)^{\frac{1}{2}}}{\left(\frac{v}{v_{c}}-1\right)^{\frac{1}{2}}+\epsilon}\right),
$$

The first term corresponds to the linear intrinsic and thermal contributions to the damping force, with a single temperature-dependent adjustable parameter $\alpha$. The term $H\left(v-v_{c}\right)$ is the Heaviside step function, which is zero when $v$ is below $v_{c}$ and switches to 1 at the onset of turbulence when $v \geq v_{c}$. The $\left(v^{2}-v_{c}{ }^{2}\right)$ factor in the second term is similar to that used by Schoepe [19] to describe oscillating sphere data, and ensures that the turbulent drag increases smoothly from zero above $v_{c}$. The parameter $\beta$ determines 


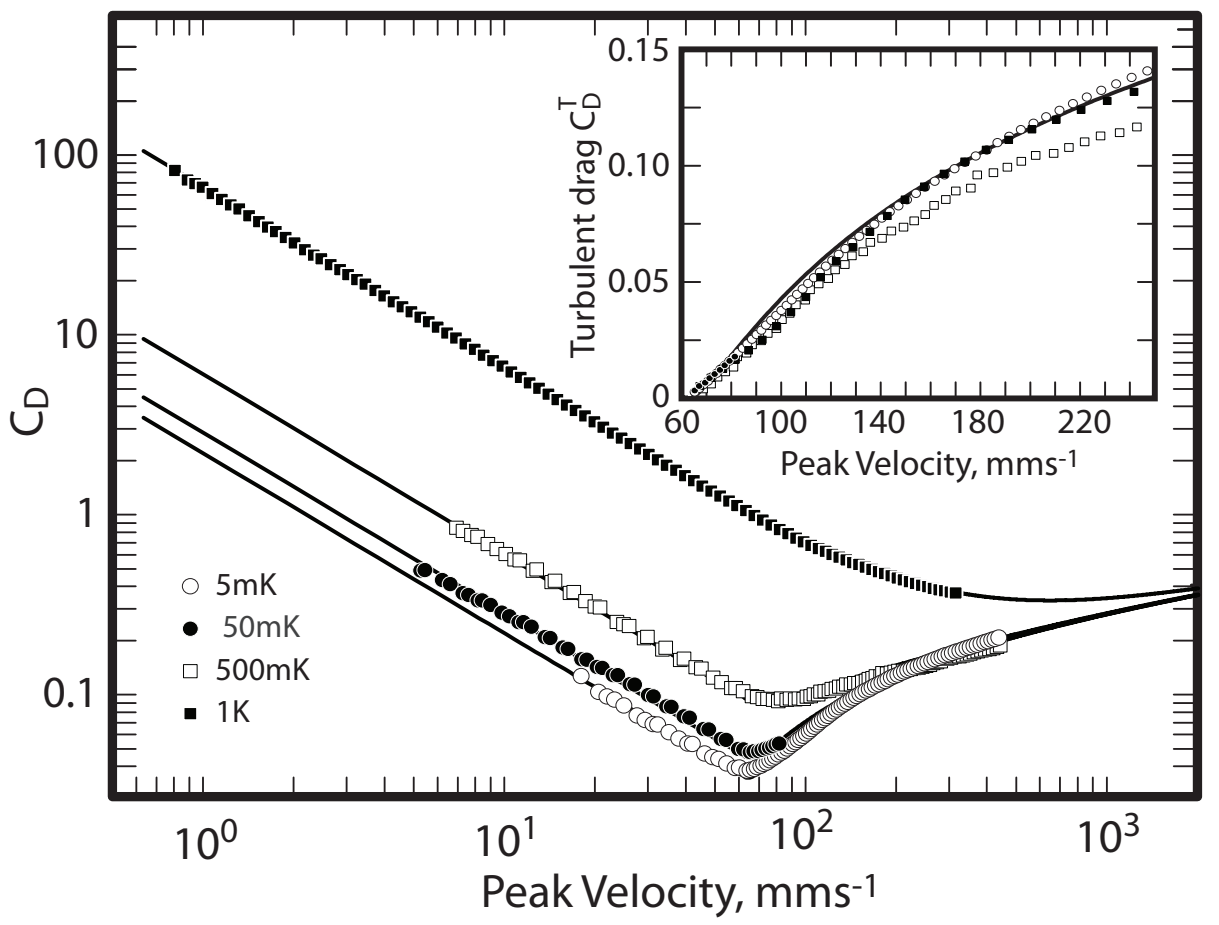

Fig. 4 The derived drag coefficient $C_{D}$ for the tuning fork as a function of velocity. Solid curves represent a fit to the data as described in the text (equation (9)). The inset shows the turbulent contribution to the drag coefficient inferred from the same data, $C_{D}^{T}$, plotted on a linear scale after subtraction of a term proportional to $v^{-1}$ representing linear damping from intrinsic properties of the fork and thermal excitations. (Note that the $50 \mathrm{mK}$ was only measured up to $85 \mathrm{mms}^{-1}$.)

the limiting value of $C_{D}$ when $v \gg v_{c}$. The final part of the second term controls the rate at which the turbulent drag develops from $v_{c}$ towards higher velocities, governed by parameter $\epsilon$. This functional form is purely empirical, and different from that chosen by the authors of [33], who have a factor $\left(v / v_{c}-1\right)^{2}$ in place of our $\left(v / v_{c}-1\right)^{\frac{1}{2}}$ and use a slightly more complicated expression involving an effective kinematic viscosity. We developed our form independently, and justify this choice (as do the authors of [33]) as giving the closest fit to our data. We are able to fit this expression to all our data at temperatures $1 \mathrm{~K}$ and below with the same values of $v_{c}, \beta$ and $\epsilon$. These fits are shown in figure 4. For all the curves we set $\beta=1, v_{c}=63 \mathrm{~mm} \mathrm{~s}^{-1}$, and $\epsilon=10$. The only parameter that separates the curves is the value of linear damping, $\alpha$, that we obtain from the linear part of $C_{D}$ at velocities below $v_{c}$. As shown figure 4 , the expression fits our data very well, and significantly better than the more complicated expression given in [33]. However, we should point out that without a well defined quantitative model the value of having such an analytical expression is somewhat limited. 


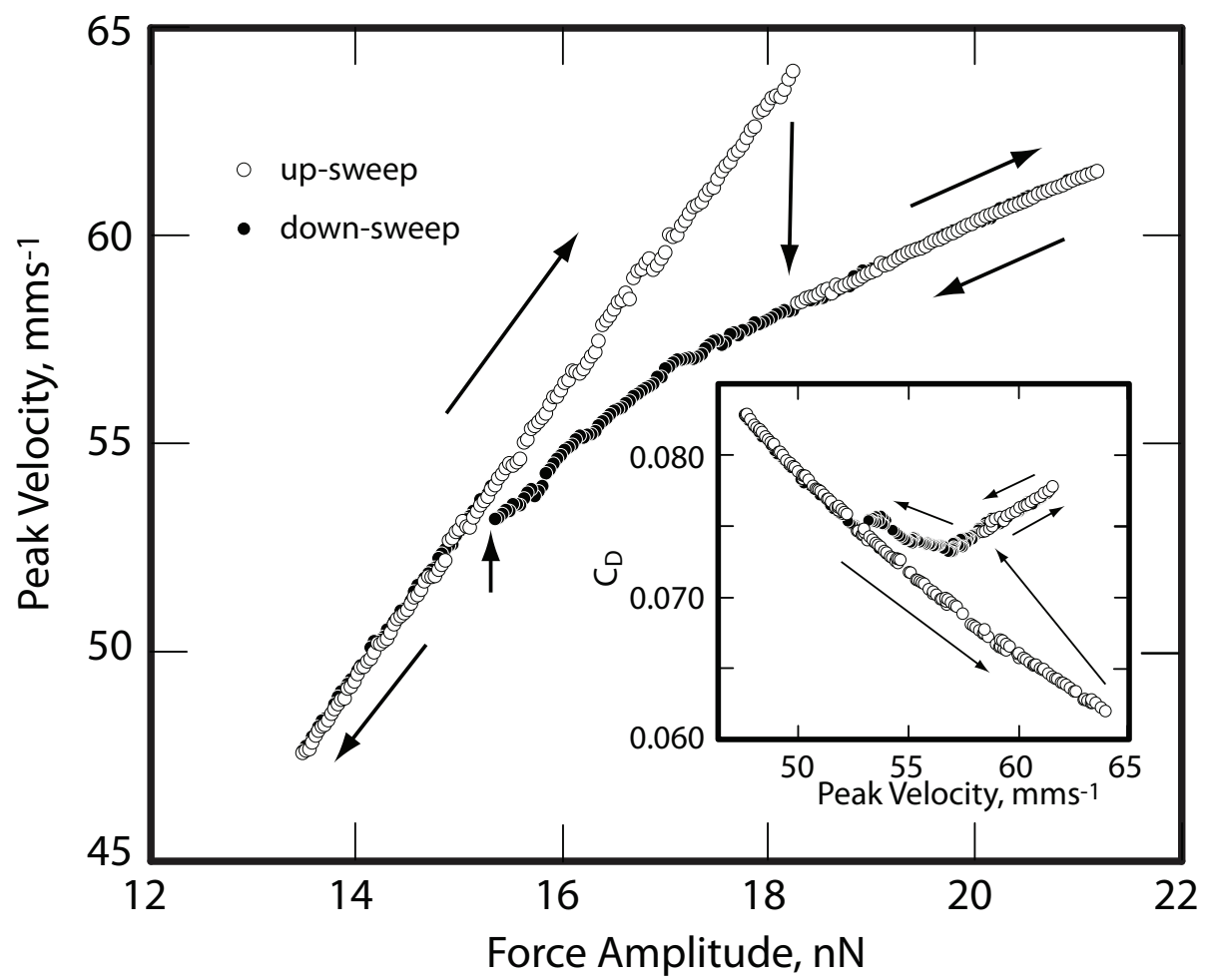

Fig. 5 The peak velocity as a function of the driving force for the fork in a later experimental cell, showing hysteresis and switching behavior, at $8 \mathrm{mK}$. Open symbols show an 'up-sweep' and closed circles show a 'down-sweep'. Inset shows drag coefficient as a function of the peak velocity.

\subsection{Switching Behaviour and Hysteresis}

In a later experiment, the same fork was placed, in the same orientation, in a new experimental cell. The behaviour of the fork was found to be qualitatively and quantitatively different, as though it was now a different object. The linear damping had increased so that Q-value at the lowest temperatures was reduced by a factor of approximately 2 , and the critical velocity for the transition to turbulence was reduced from 63 to $53 \mathrm{~mm} \mathrm{~s}^{-1}$, making comparison with previous results problematic. We do not know why the properties changed, but can speculate that the different behaviour may be due to dirt on the tuning fork prongs. This irreproducibility of fork behaviour may limit their use as reliable cryogenic tools at the lowest temperatures.

However, measurements in the new cell now revealed switching between the laminar and turbulent regimes and hysteresis, allowing for comparison with similar observations made previously with wire resonators $[1,2,6-11]$ and oscillating spheres [19-22]. A typical response curve, taken at $8 \mathrm{mK}$ and 1 bar pressure, is shown in figure 5 . The inset to figure 5 shows the corresponding drag coefficient as a function of the velocity. As the driving force is increased, the velocity increases into a metastable laminar flow state, before stepping down, switching into the turbulent flow state. As the drive is 


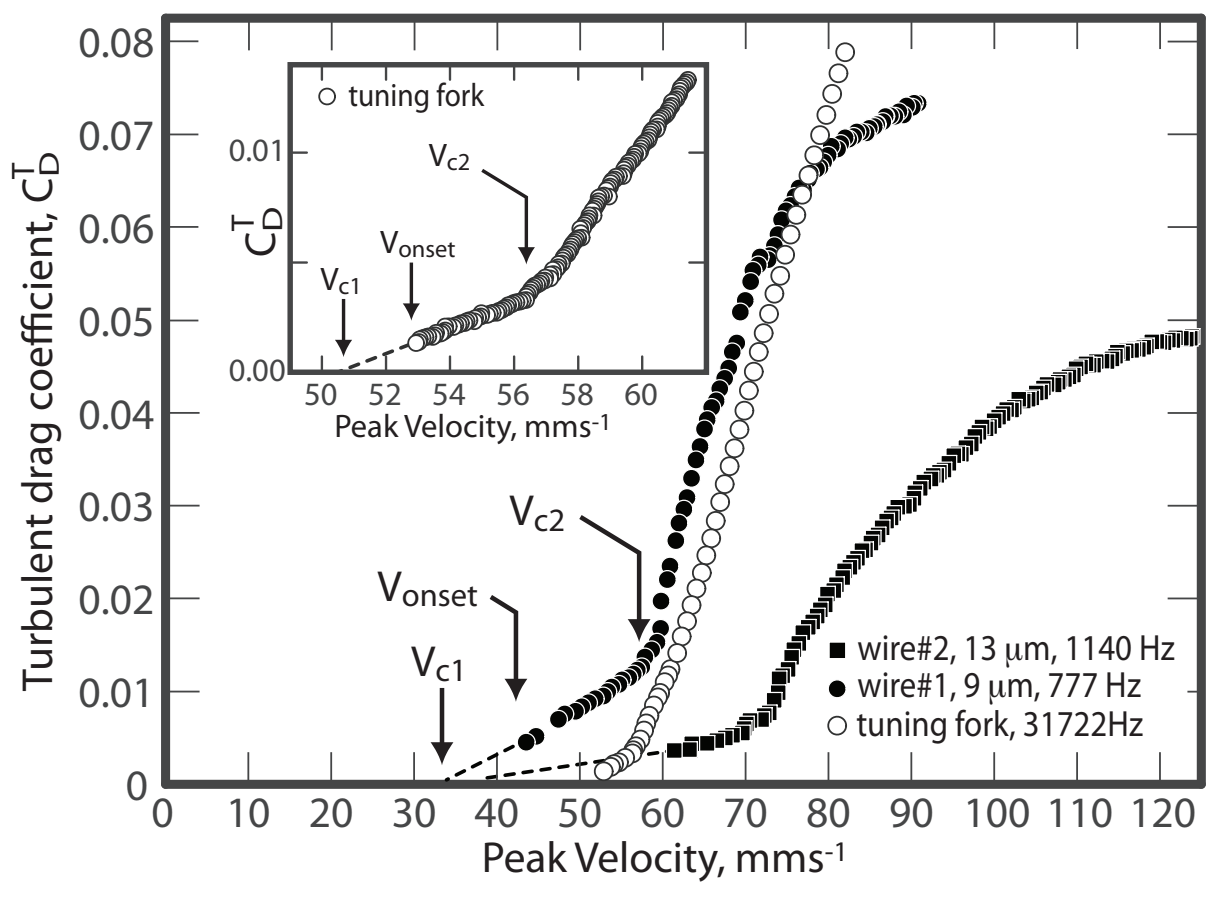

Fig. 6 The turbulent contribution to the drag coefficient as a function of peak velocity for the tuning fork, compared with that for two vibrating wire resonators [2]. The inset shows a close-up of the tuning fork data. The data were taken whilst decreasing the drive amplitude.

decreased again, the velocity decreases continuously back down towards the laminar flow region, often (as in figure 5) showing a small step up in velocity as the laminar regime is rejoined.

\subsection{Comparison with Vibrating Wires}

We can compare the observed behaviour of the fork directly with that measured for vibrating wires reported in [2]. In figure 6 we plot the turbulent contribution to the drag coefficient as a function of peak velocity for our fork, together with the earlier data for two vibrating wires of diameters 9 and $13 \mu \mathrm{m}$. The inset shows an expanded view of the behaviour in the transition region. For each device, we show a down sweep, i.e. the driving force is reducing during the sweep. This allow us to measure the turbulent drag coefficient down to lower velocities since the transition to turbulence occurs at a lower velocity on a down sweep. The similarities between wire and fork, as revealed in figure 6, are quite remarkable:

(i) We observe a velocity, $v_{\text {onset }}$, above which extra turbulent drag exists. For each device, this is highly irreproducible from one sweep to the next. The up sweeps give a higher and again irreproducible onset velocity. We also have strong evidence that 
the onset velocity is reduced in the presence of surrounding vorticity produced from a neighbouring device [1]. Further measurements to better quantify this for the fork are ongoing.

(ii) For each device, we can clearly define a critical velocity $v_{c 1}$ by extrapolating to zero turbulent drag. This velocity is reproducible between sweeps and corresponds to the minimum velocity needed to produce and sustain the vortex lines that create drag. (iii) For each device, we can identify another reproducible critical velocity $v_{c 2}$ above which the drag rises much more steeply.

(iv) Not only do we observe the same features, but the magnitudes of the three characteristic velocities are very similar for the two wires and the fork. Furthermore, the initial rise in the turbulent drag coefficient has a similar magnitude to that of the two wire resonators. This is surprising given the large differences in the geometries of the devices and their different resonant frequencies (we note that the vibrating wire response was found to have a weak frequency dependence [2]). However, compared to the fork, the turbulent drag on the vibrating wires appears to saturate at much lower values and at much lower velocities.

\subsection{The Superfluid Inertial Coefficient of a Tuning Fork}

Figure 7 shows the resonant frequency of the fork as a function of velocity. The vacuum data were taken at the base temperature of the dilution refrigerator before any helium had been let into the cell. In this case the temperature of the fork is necessarily uncertain, having cooled via its electrical leads that are thermally anchored to the mixing chamber and to the cell body. As shown in the figure, there is a small decrease in the resonant frequency, on the order of $0.15 \mathrm{~Hz}$, dropping more quickly as the velocity increases. The variation indicates that the effective mass and/or spring constant of the fork must have some dependence on the amplitude of its motion.

When the fork is immersed in helium, its resonant frequency at low amplitude drops below that in vacuum due to the added mass of fluid back-flow (corresponding to the inertial coefficient of the fluid flow). The $500 \mathrm{mK}$ data set of Fig. 7 is representative of all the superfluid data, showing a further drop in frequency of about $0.2 \mathrm{~Hz}$ as the velocity increases, roughly comparable with that of the fork in vacuum. We believe that the variation is heavily dominated by the intrinsic properties of the fork. The frequency changes shown in Fig. 7 are very similar in vacuum and in the superfluid. The small differences are likely to be due to the differences in the surface cleanliness of the fork and/or due the fact that the temperature of the fork for the vacuum measurements is ill-defined owing to the lack of good thermal contact.

At $500 \mathrm{mK}$ and 1 bar pressure the helium density is $146 \mathrm{~kg} \mathrm{~m}^{-3}$ and the resonant frequency of the fork has dropped by $877 \mathrm{~Hz}$ below that in vacuum, giving $C_{M}=0.98$. This is very close to 1 , and is similar to values which were previously measured for vibrating wire resonators [1].

The inset to the figure 7 shows an expanded view of the small changes in the resonant frequency observed around the transition region. The data shown are for an up sweep, in which the driving force is slowly incremented to higher values. The inset shows the resonant frequency decreasing with increasing velocity in the laminar regime (open symbols), the velocity then steps down and the frequency steps up a little as the fork enters the turbulent regime (closed symbols). Despite the frequency increase as the 


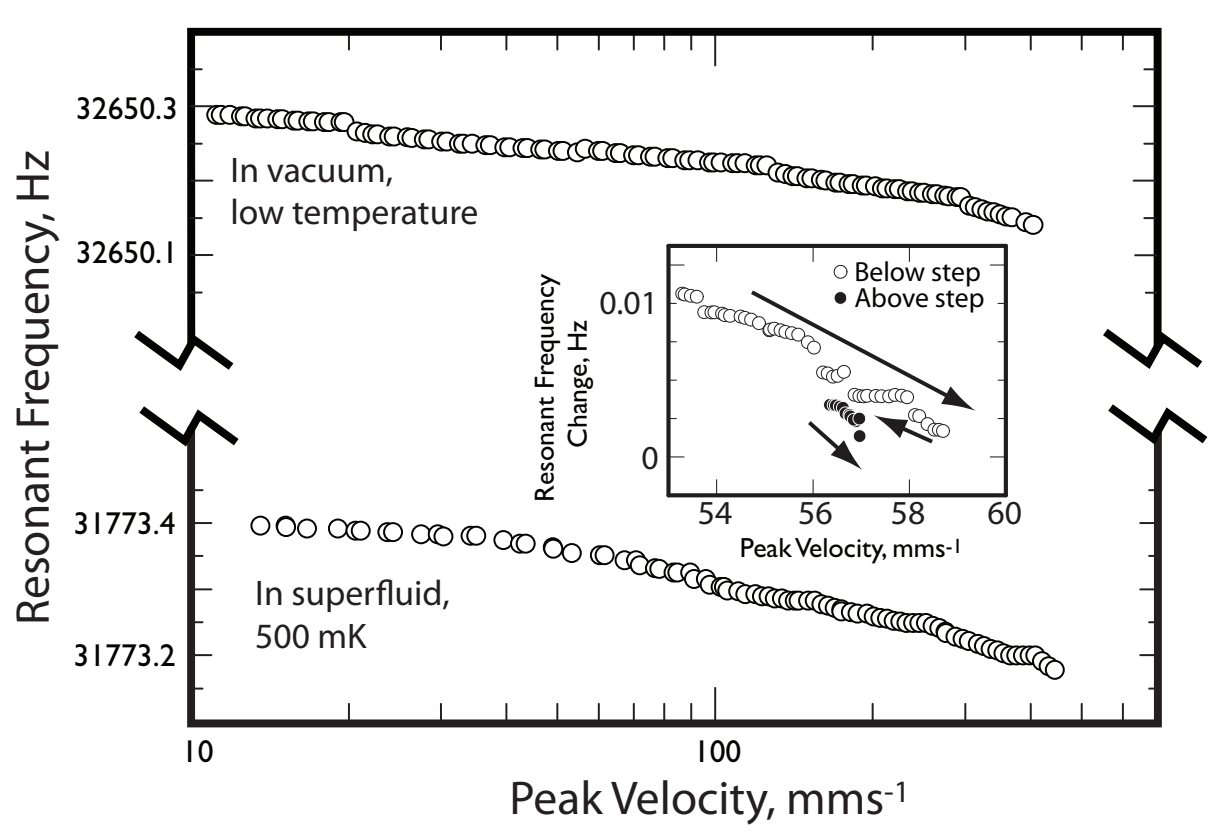

Fig. 7 The resonant frequency of the fork as a function of peak velocity. Upper data show the intrinsic response in vacuum with the dilution refrigerator at $5 \mathrm{mK}$. Lower data show the fork response in superfluid ${ }^{4} \mathrm{He}$ at $500 \mathrm{mK}$ and 1 bar pressure. The small steps on the vacuum data are due to small phase changes or "relay clicks" as the function generator switches range. This effect has been largely accounted for and removed in the ${ }^{4} \mathrm{He}$ data. The inset shows a close-up of the small frequency changes observed on sweeping up the fork driving force from the laminar(open symbols) to the turbulent (closed symbols) regime. Note the velocity steps down during the transition.

turbulent regime is entered, the resonant frequencies in the turbulent regime, at a given velocity, are found to be very slightly lower than in the laminar regime. This behaviour is the main qualitative difference between forks and vibrating wire resonators.

For the vibrating wires, the frequency is significantly higher in the turbulent regime compared to the laminar regime. We can express this frequency shift as a change in the inertial coefficient, $C_{M}$. When a wire produces turbulence [2], the decrease of the inertial coefficient $\delta C_{M}$ in the turbulent regime is found to have a magnitude very similar to the turbulent drag coefficient, i.e. $\delta C_{M} \simeq C_{D}$. This is clearly not the case for the fork. Since the frequency shift is negative in the turbulent state, the inertial coefficient actually rises. Also the change is extremely small, at least a thousand times smaller (and of opposite sign) than that observed with vibrating wires. For the wires it is argued that vortex shedding leads to a reduction in backflow and hence reduced inertial mass [2]. The fork operates at much higher frequency than the wires, and one tentative explanation of the difference in behaviour is that vortices in the higher frequency flow field are unable to respond on the timescale of the fork motion and are therefore unable to affect the inertia of the superfluid backflow. This reasoning was first suggested to explain the slight frequency dependence of the inertial coefficient observed in vibrating wire measurements [2]. 


\section{Conclusions}

We have measured the transition to turbulence of a resonating quartz tuning fork immersed in superfluid ${ }^{4} \mathrm{He}$ over a wide range of temperatures and pressures, concentrating on its behaviour in the low temperature, pure superfluid limit. We have shown that the transition from laminar to turbulent flow is marked by the sudden appearance of excess non-linear damping on the fork motion. The excess damping at low temperatures is insensitive to pressure. The drag coefficient that we infer from our data is broadly consistent with that measured by other groups, and the turbulent drag fits well to a simple empirical formula. We find that the turbulent drag is temperature independent below $1 \mathrm{~K}$.

In a new experimental cell the behaviour of the same fork was found to have changed. Now we observed metastability of the laminar regime, and consequent hysteresis and switching behaviour in the fork response. The behavior around the turbulent transition is remarkably similar to that observed for vibrating wires. However there is one major qualitative difference. For vibrating wires, the inertial coefficient decreases substantially in the turbulent regime, while for the fork the inertial coefficient increases but by a very small amount. Work is in progress to characterise more fully the switching behaviour and its potential use to detect turbulence from other sources.

\section{Acknowledgements}

We are grateful to S.M. Holt, A.M. Stokes and M.G. Ward for their technical support. We also thank V. Efimov, D. Garg, R. Hänninen, W. Schoepe, G.A. Sheshin, L. Skrbek, P. Skyba, W.F. Vinen and H. Yano for useful discussions. We acknowledge the financial support of the U.K. EPSRC and the Royal Society.

\section{References}

1. D.I. Bradley, D.O. Clubb, S.N. Fisher, A.M. Guénault, R.P. Haley, C.J. Matthews, G.R. Pickett and K.L. Zaki, J. Low Temp. Phys. 138, 493 (2005).

2. D.I. Bradley, S.N. Fisher, A.M. Guénault, R.P. Haley, V. Tsepelin, G.R. Pickett and K.L. Zaki, J. Low Temp. Phys. 154, 97 (2009).

3. D.I. Bradley, Phys. Rev. Lett. 84, 1252 (2000).

4. S.N. Fisher, A.J. Hale, A.M. Guénault and G.R. Pickett, Phys. Rev. Lett. 86, 244 (2001).

5. D.I. Bradley, S.N. Fisher, A.M. Guénault, M.R. Lowe, G.R. Pickett, A. Rahm and R.C.V. Whitehead, Phys. Rev. Lett. 93, 235302 (2004).

6. H. Yano, A. Handa, H. Nakagawa, K. Obara, O. Ishikawa, T. Hara and M. Nakagawa, J. Low Temp. Phys. 138, 561 (2005).

7. H. Yano, A. Handa, H. Nakagawa, K. Obara, O. Ishikawa and T. Hata, J. Phys. Chem. Solids 66, 1501 (2005)

8. H. Yano, N. Hashimoto, A. Handa, M. Nakagawa, K. Obara, O. Ishikawa and T. Hata, Phys. Rev. B 75, 012502 (2007).

9. N. Hashimoto, R. Goto, H. Yano, K. Obara, O. Ishikawa and T. Hata, Phys. Rev. B 76, 020504 (2007).

10. N. Hashimoto, A. Handa, M. Nakagawa, K. Obara, H. Yano, O. Ishikawa and T. Hata, J. Low Temp. Phys. 148, 299 (2007).

11. R. Goto, S. Fujiyama, H. Yano, Y. Nago, N. Hashimoto, K. Obara, O. Ishikawa, M. Tsubota and T. Hata, Phys. Rev. Lett. 100, 045301 (2008).

12. J. Martikainen, J. Tuoriniemi, T. Knuuttila and G.R. Pickett, J. Low Temp. Phys. 126, 139 (2002). 
13. D.I. Bradley, D.O. Clubb, S.N. Fisher, A.M. Guénault, C.J. Matthews and G.R. Pickett, J. Low Temp. Phys. 134, 381 (2004).

14. D.I. Bradley, D.O. Clubb, S.N. Fisher, A.M. Guénault, R.P. Haley, C.J. Matthews, G.R. Pickett, V. Tsepelin and K. Zaki Phys. Rev. Lett. 95, 035302 (2005).

15. D.I. Bradley, D.O. Clubb, S.N. Fisher, A.M. Guénault, R.P. Haley, C.J. Matthews, G.R. Pickett, V. Tsepelin and K.L. Zaki, Phys. Rev. Lett. 96, 035301 (2006).

16. D.I. Bradley, S.N. Fisher, A.M. Guénault, R.P. Haley, N. Mulders, S. O’Sullivan, G.R. Pickett, J. Roberts and V. Tsepelin, Phys. Rev. Lett. 10165302 (2008).

17. H.A. Nichol, L. Skrbek, P.C. Hendry and P.V.E. McClintock, Phys. Rev. E 70, 056307 (2004).

18. D. Charalambous, L. Skrbek, P.C. Hendry, P.V.E. McClintock and W.F. Vinen, Phys. Rev. E 74, 036307 (2006).

19. J. Jäger, B. Schuderer and W. Schoepe, Phys. Rev. Lett. 74, 566 (1995).

20. M. Niemetz, H. Kerscher and W. Schoepe, J. Low Temp. Phys. 126, 287 (2002).

21. M. Niemetz and W. Schoepe, J. Low Temp. Phys. 135, 447 (2004).

22. W. Schoepe, Phys. Rev. Lett. 92, 095301 (2004).

23. R. Hänninen and W. Schoepe, J. Low Temp. Phys. 153, 189 (2008).

24. W. Schoepe, J. Low Temp. Phys. 150, 724 (2008)

25. J. Luzuriaga, J. Low Temp. Phys. 108, 561 (1997)

26. R. Blaauwgeers, M. Blažková, M. C̆lovečko, V.B. Eltsov, R. de Graaf, J. Hosio, M. Krusius, D. Schmoranzer, W. Schoepe, L. Skrbek, P. Skyba, R.E. Solntsev and D.E. Zmeev, J. Low Temp. Phys. 146, 537 (2007).

27. M. Blažková, M. Clovečko, E. Gažo, L. Skrbek, P. Skyba, J. Low Temp. Phys. 148, 305 (2007).

28. M. Blažková, D. Schmoranzer, L. Skrbek, Phys. Rev. E 75, 025302 (2007).

29. M. Blažková, M. C̆lovečko, V.B. Eltsov, E. Gažo, R. de Graaf, J.J. Hosio, M. Krusius, D. Schmoranzer, W. Schoepe, L. Skrbek, P. Skyba, R.E. Solntsev and W.F. Vinen, J. Low Temp. Phys. 150, 525 (2008).

30. M. Blažková, T.V. Chagovets, M. Rotter, D. Schmoranzer and L. Skrbek, J. Low Temp. Phys. 150, 194 (2008).

31. G.A. Sheshin, A.A. Zadorozhko, E.Y. Rudavskii, V.K. Chagovets, L. Skrbek and M. Blažková, Low Temp. Phys 34, 875 (2008).

32. E.M. Pentti, J.T. Tuoriniemi, A.J. Salmela, A.P. Sebedash, J. Low Temp. Phys. 150, 555 (2008).

33. M. Blažková, D. Schmoranzer, L. Skrbek and W. F. Vinen, Phys. Rev. B 79, 054522 (2009).

34. S.N. Fisher, A.M. Guénault, C.J. Kennedy and G.R.Pickett, Phys. Rev. Lett. 63, 2566 (1989).

35. S.N. Fisher, G.R. Pickett, and R.J. Watts-Tobin J. Low Temp. Phys. 83225 (1991).

36. M.P. Enrico, S.N. Fisher and R.J. Watts-Tobin, J. Low Temp. Phys. 98, 81 (1995).

37. C. Bauerle, Y.M. Bunkov, S.N. Fisher and H. Godfrin, Phys. Rev. B. 57, 14381 (1998).

38. D.O. Clubb, O.V.L. Buu, R.M. Bowley, R. Nyman and J.R. Owers-Bradley, J. Low Temp. Phys 136, 1 (2004).

39. M.Tsubota, T. Araki and S.K. Nemirovskii, Phys. Rev. B 62, 11751 (2000).

40. D. Kivotides, J.C. Vassilicos, D.C. Samuels and C.F. Barenghi, Phys. Rev. Lett. 86, 3080 (2001).

41. T. Araki, M. Tsubota and S.K. Nemirovskii, Phys. Rev. Lett. 89, 145301 (2002).

42. W.F. Vinen, M. Tsubota and A. Mitani, Phys. Rev. Lett. 91, 135301 (2003).

43. M. Tsubota and M. Kobayashi, AIP Conference Proceedings 850, 219 (2006).

44. R. Hänninen. M. Tsubota and W.F. Vinen, Phys. Rev. B 75, 064502 (2007).

45. S. Fujiyama and M. Tsubota, Phys. Rev. B 79, 094513 (2009).

46. W.F. Vinen, Phys. Rev. B 71, 024513 (2005).

47. C.F. Barenghi and D.C. Samuels, Phys. Rev. Lett. 89, 155302 (2002).

48. W.F. Vinen, Phys. Rev. B. 71, 024513 (2005).

49. C.F. Barenghi, Y.A. Sergeev, and N. Suramlishvili, Phys. Rev. B 77, 104512 (2008).

50. P.E. Roche and C.F. Barenghi, Europhys. Lett. 81, 36002 (2008).

51. W.F. Vinen and J.J. Niemela, J. Low Temp. Phys. 128, 167 (2002).

52. "Quantum Turbulence", Progress in Low Temperature Physics, Vol. 16, Elsevier B.V., editors M. Tsubota and W.P. Halperin, (2009).

53. Quartz Tuning Forks, part label "KDS5M", from KDS America / Daishinku Corporation.

54. P. Skyba and S.M. Holt, private communication, and to be published. 
55. K. Karrai, lecture notes (2000) at http://www.nano.physik.unimuenchen.de/publikationen/Preprints/p-00-03_Karrai.pdf

56. T. Sarpkaya, J. Fluid Mech. 165, 61 (1986).

57. J.R. Morison, M.P. O'Brien, J.W. Johnson and S.A. Schaaf, Trans American Inst. of Mining and Metall. Eng. 189, 149 (1950).

58. C.H.K. Williamson, J. Fluid Mech. 155, 141 (1985). 